\title{
Numerical Method for Non-Linear Conservation Laws: Inviscid Burgers Equation
}

\author{
R. Hemel, M. T. Azam, M. S. Alam \\ Department of Mathematics, Jahangirnagar University, Dhaka, Bangladesh \\ Email: hemel07.ju@gmail.com
}

How to cite this paper: Hemel, R., Azam, M.T. and Alam, M.S. (2021) Numerical Method for Non-Linear Conservation Laws: Inviscid Burgers Equation. Journal of Applied Mathematics and Physics, 9, 13511363.

https://doi.org/10.4236/jamp.2021.96092

Received: April 21, 2021

Accepted: June 26, 2021

Published: June 29, 2021

Copyright $\odot 2021$ by author(s) and Scientific Research Publishing Inc. This work is licensed under the Creative Commons Attribution International License (CC BY 4.0).

http://creativecommons.org/licenses/by/4.0/

\begin{abstract}
This paper deals with the Burgers equation which is the most common model used in the nonlinear conservation laws. Here the theoretical aspect of conservation law is discussed by using inviscid Burgers equation. At first, we introduce the general non-linear conservation law as a partial differential equation and its solution procedure by the method of characteristic. Next, we present the weak solution of the problem with entropy condition. Taking into account shock wave and rarefaction wave, the Riemann problem has also been discussed. Finally, the finite volume method is considered to approximate the numerical solution of the inviscid Burgers equation with continuous and discontinuous initial data. An illustration of the problem is provided by some examples. Moreover, the Godunov method provides a good approximation for the problem.
\end{abstract}

\section{Keywords}

Conservation Law, Inviscid Burgers Equation, Shock Wave, Rarefaction Wave, Finite Volume Method

\section{Introduction}

The Nonlinear conservation law is used to describe many physical phenomena mathematically, and numerical methods are required to solve these physical problems [1] [2] [3] [4]. The governing one-dimensional conservation law takes the form [5],

$$
\frac{\partial u}{\partial t}+\frac{\partial}{\partial x} f(u)=0, t>0, x \in R
$$

where $f(u)$ is the flux function. The quantity $u$ is the density of some fluid and $\int u(x, t) \mathrm{d} x$ treated as mass of the fluid which is conserved in the conservation 
laws. In the paper, we will discuss integral form of conservation law, classical solution and weak solution for understanding the theoretical knowledge of the problem. To understand the shock wave and rarefaction wave, The Riemann problem also has been discussed. Finally, finite volume method is considered to approximate the numerical solution of the problem.

In finite difference method, the derivatives are approximated by finite differences and therefore, the differential equation does not hold near the discontinuities in the solution. The differential form of the conservation law may not be applied when the shock is generated. After shock formulation, shock actually travels with shock speed [6]. In this paper, finite volume method is considered, which is based on the integral form instead of differential equation. In this method, the domain is divided into grid cells and approximates the total integral of density, $u$ over each grid cell average of density, $u$, which is the integral divided by the volume of the cell. On the other hand, in finite difference method, the pointwise approximation is needed at the grid point. The cell average is changed in each time step by the flux through the edges of the grid cell. Therefore, it is important to choose a good numerical flux function that approximates the correct fluxes reasonably well [5]. Therefore finite volume method is effective to describe the discontinuous solution. The main objective of this paper is to approximate the numerical solution of the non-linear conservation law by using the inviscid Burgers equation. In this paper, Godunov method is considered to approximate the numerical solution of the problem with continuous and discontinuous initial data. The Godunov method is a conservative numerical method, which is used for the solution of hyperbolic conservation laws. This method can be characterized by the solution (exact or approximate) of a Riemann problem within computational cells in order to obtain numerical fluxes. This method is first-order accurate in both space and time.

\section{Integral Form of Conservation Laws}

The integral form of the conservation law is obtained by integrating (1) within the interval $\left[x_{1}, x_{2}\right]$,

$$
\frac{\mathrm{d}}{\mathrm{d} t} \int_{x_{1}}^{x_{2}} u(x, t) \mathrm{d} x+f\left(u\left(x_{2}, t\right)\right)-f\left(u\left(x_{1}, t\right)\right)=0
$$

Suppose is a conserved quantity in $\left[x_{1}, x_{2}\right]$ and defined as, $Q(t)=\int_{x_{1}}^{x_{2}} u(x, t) \mathrm{d} x$ then,

$$
\frac{\mathrm{d}}{\mathrm{d} t} Q(t)=f\left(u\left(x_{1}, t\right)\right)-f\left(u\left(x_{2}, t\right)\right)
$$

Again, integrate the above equation over time $[0, T]$,

$$
Q(T)-Q(0)+\int_{0}^{T}\left[f\left(u\left(x_{1}, t\right)\right)-f\left(u\left(x_{2}, t\right)\right)\right] \mathrm{d} t=0
$$

The above equation is the integral form of the conservation law which directly interpreted that the difference in the total amount of the state variable $u$ in $\left[x_{1}, x_{2}\right]$ between in a specific time is equal to the difference in the total flux 
through the boundary integrated over that time duration [6].

\section{Classical Solution}

To understand the classical solution, initially we will consider simple linear conservation law, known as linear advection equation [6] where $f(u)=a u$,

$$
\frac{\partial u}{\partial t}+a \frac{\partial u}{\partial x}=0 ; u(x, 0)=f\left(x_{0}\right)
$$

The solution of advection equation can be easily derived from the concept of method of characteristic. Here, the characteristic curve $\frac{\mathrm{d} x(t)}{\mathrm{d} t}=a$, describe the straight line with slope $a$ in $x=x(t)$ curve and the solution is

$$
u(x, t)=f(x-a t)
$$

Thus, linear conservation law transport the initial data in the $x$-direction and also it is exactly as smooth as the initial data.

Now we will consider a simple non-linear conservation laws, in particular, Burgers equation. The study of Burgers equation which is in the class of nonlinear conservation law has been continued to draw the attention of researchers as it represents various physical phenomenon such as theory of shock wave, rarefaction wave, dynamics of fluid or gas etc. [7] [8] [9] [10]. The classical Burgers equation is described by,

$$
\frac{\partial u}{\partial t}+\frac{\partial}{\partial x}\left(\frac{u^{2}}{2}\right)=v \frac{\partial^{2} u}{\partial x^{2}}
$$

In this research, Burgers equation with zero viscosity term is considered which is known as inviscid Burgers equation. The inviscid Burgers equation is a basic case study as it has also the properties of nonlinear conservation law [11]. Therefore inviscid Burgers equation takes the form,

$$
\frac{\partial u}{\partial t}+\frac{\partial}{\partial x}\left(\frac{u^{2}}{2}\right)=0 ; u(x, 0)=f\left(x_{0}\right)=u\left(x_{0}\right)
$$

where flus function is, $f(u)=\frac{1}{2} u^{2}$. The characteristic curve of the equation is

$$
\frac{\mathrm{d} x(t)}{\mathrm{d} t}=u(x(t), t)
$$

The characteristics are straight lines with slope $u$, and the solution is

$$
u(x, t)=f(x-u t)=u_{0}(x-u t)
$$

Thus, the solution is constant along the characteristic curves. In this case, discontinuity may form smooth initial conditions in finite time. Therefore, the study of solution of Burgers equation allows interesting phenomena such as shock waves and rarefaction waves.

\section{Weak Solution}

In classical sense, if $u$ be a piecewise constant solution of the conservation law 
(1) and also discontinuous along the characteristic curve with any shock speed (s) then $u$ is a weak solution of (1). The shock speed can be derived from the Rankine-Hugoniot jump condition [5] [12]. To get the shock speed, we need to integrate (1) in rectangle $\left[x_{1}, x_{1}+\Delta x\right] \times\left[t_{1}, t_{1}+\Delta t\right]$,

$$
\begin{aligned}
& \int_{x_{1}}^{x_{1}+\Delta x} u\left(x, t_{1}+\Delta t\right) \mathrm{d} x-\int_{x_{1}}^{x_{1}+\Delta x} u\left(x, t_{1}\right) \mathrm{d} x \\
& =\int_{t_{1}}^{t_{1}+\Delta t} f\left(u\left(x_{1}, t\right)\right) \mathrm{d} t-\int_{t_{1}}^{t_{1}+\Delta t} f\left(u\left(x_{1}+\Delta x, t\right)\right) \mathrm{d} t
\end{aligned}
$$

Suppose shock splits the rectangle into two parts and $u_{L}$ and $u_{R}$ are two states immediately to the left and right side of the shock, then

$$
\Delta x u_{R}-\Delta x u_{L}=\Delta t f\left(u_{L}\right)-\Delta t f\left(u_{R}\right)
$$

If $\Delta x=-s \Delta t$, for $s<0$ and then dividing by $\Delta t$ and taking $\Delta t \rightarrow 0$ gives,

$$
\begin{gathered}
s\left(u_{R}-u_{L}\right)=f\left(u_{R}\right)-f\left(u_{L}\right) \\
s=\frac{f\left(u_{R}\right)-f\left(u_{L}\right)}{u_{R}-u_{L}}
\end{gathered}
$$

Which is known as the Rankine-Hugoniot Condition and $s$ is the shock speed.

The shock speed for Inviscid Burgers equation is

$$
s=\frac{1}{2}\left(u_{L}+u_{R}\right), \text { as } f(u)=\frac{1}{2} u^{2} .
$$

Therefore it is easy to calculate the shock speed. In general, weak solutions occur whenever there is no smooth or classical solution and these solutions may not be differential or even continuous. Therefore, in this case, Equation (1) is not valid and the integral form of conservation law does hold. In mathematically, the function $u(x, t)$ is a weak solution of conservative law (1) with initial condition if it satisfies the following, for all function of $\varphi(x, t)$ [13],

$$
\int_{0}^{\infty} \int_{-\infty}^{\infty}\left[u \varphi_{t}+f(u) \varphi_{x}\right] \mathrm{d} x \mathrm{~d} t=-\int_{0}^{\infty} u(x, 0) \varphi(x, 0) \mathrm{d} x
$$

However, the weak solution concept cannot guarantee the uniqueness of the solution, because of that addition condition need to be imposed, called entropy condition.

\section{Entropy Condition}

The entropy condition described by the following [12],

If a discontinuity propagate with the characteristic speed $s$, given by the Rankine-Hugoniot condition, satisfies the entropy condition if,

$$
f^{\prime}\left(u_{L}\right)>s>f^{\prime}\left(u_{R}\right)
$$

In particular, for the inviscid Burgers equation, if $u$ satisfy the entropy condition then,

$$
u_{L}>u_{R}
$$

where $f(u)=\frac{1}{2} u^{2}$. 


\section{Riemann Problem}

The Riemann problem is a particular initial value problem which contains a conservation law together with piecewise constant data having a single discontinuity. We consider the Riemann problems for the inviscid Burgers equation subject to special initial conditions, described by

$$
u(x, 0)= \begin{cases}u_{L} & x<0 \\ u_{R} & x>0\end{cases}
$$

The solution of the Riemann problem depends on $u_{L}$ and $u_{R}$. If $u_{L}>u_{R}$ then the characteristic curve is,

$$
x_{0}=x+u_{L} t \text { if } x<0 \text { and } x_{0}=x+u_{R} t \text { if } x>0
$$

Then the solution becomes,

$$
u(x, t)= \begin{cases}u_{L} & x-s t<0 \\ u_{R} & x-s t>0\end{cases}
$$

Figure 1 shows the characteristic curve for $u_{L}>u_{R}$. Therefore, for $u_{L}>u_{R}$, left wave travel faster than the right wave and as a result, a shock wave is generate. In this case, the solution satisfies the entropy condition also.

On the other hand, if $u_{L}<u_{R}$, the problem has two solutions. First one is,

$$
u(x, t)= \begin{cases}u_{L} & x-s t<0 \\ u_{R} & x-s t>0\end{cases}
$$

But this solution does not follow the entropy condition, which is described above [12].

Another solution is weak solution. To get the weak solution suppose $u(x, t)=\varphi(x / t)$, then the problem becomes,

$$
\begin{gathered}
-\frac{x}{t^{2}} \varphi^{\prime}\left(\frac{x}{t}\right)+\frac{1}{t} \varphi^{\prime}\left(\frac{x}{t}\right) f^{\prime}\left(\varphi\left(\frac{x}{t}\right)\right)=0 \\
f^{\prime}\left(\varphi\left(\frac{x}{t}\right)\right)=\frac{x}{t} \\
\varphi\left(\frac{x}{t}\right)=\left(f^{\prime}\right)^{-1}\left(\frac{x}{t}\right)
\end{gathered}
$$

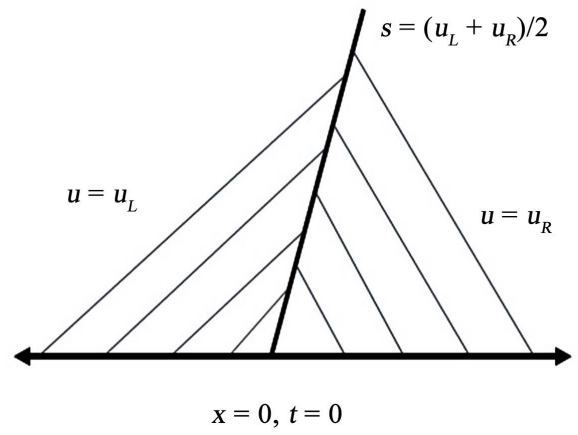

Figure 1. Characteristic curves for shock wave $u_{L}>u_{R}$. 
For $u(x, t)=\varphi\left(\frac{x}{t}\right)=u_{L}$, that is, $f^{\prime}\left(u_{L}\right)=\frac{x}{t}$

$$
\varphi\left(\frac{x}{t}\right)=\varphi\left(f^{\prime}\left(u_{L}\right)\right)=\left(f^{\prime}\right)^{-1}\left(f^{\prime}\left(u_{L}\right)\right)=u_{L} \text { if } \frac{x}{t}<f^{\prime}\left(u_{L}\right)
$$

On the other hand, $u(x, t)=\varphi\left(\frac{x}{t}\right)=u_{R}$

$$
\varphi\left(\frac{x}{t}\right)=\varphi\left(f^{\prime}\left(u_{R}\right)\right)=\left(f^{\prime}\right)^{-1}\left(f^{\prime}\left(u_{R}\right)\right)=u_{R} \text { if } f^{\prime}\left(u_{R}\right)<\frac{x}{t}
$$

Therefore, weak solution becomes,

$$
u(x, t)= \begin{cases}u_{L} & \text { if } \frac{x}{t}<f^{\prime}\left(u_{L}\right) \\ \frac{x}{t} & \text { if } f^{\prime}\left(u_{L}\right)<\frac{x}{t}<f^{\prime}\left(u_{R}\right) \\ u_{R} & \text { if } f^{\prime}\left(u_{R}\right)<\frac{x}{t}\end{cases}
$$

Thus, if $u_{L}<u_{R}$, the characteristic waves are spreading and produced rarefaction wave, head of the wave is faster than tail. Figure 2 shows the rarefaction wave.

The study of Riemann problem is very useful for understanding shock wave and rarefaction wave which appear as characteristics in the solution. In numerical methods, Riemann problem is also the basic tool for developing finite volume methods for the solution of conservation law equations due to the discreteness of the grid.

The Riemann problem has a similarity solution, a function of $x / t$ alone; selfsimilar at different times. The solution $u(x, t)=\tilde{u}(x / t)$ is constant along any ray $x / t=$ constant through the origin.

\section{Numerical Method}

In this paper, finite volume method is considered for the numerical solution of the one dimensional conservation law. Initially, we have discussed the discretization the computational domain in both space and time of the problem (1).

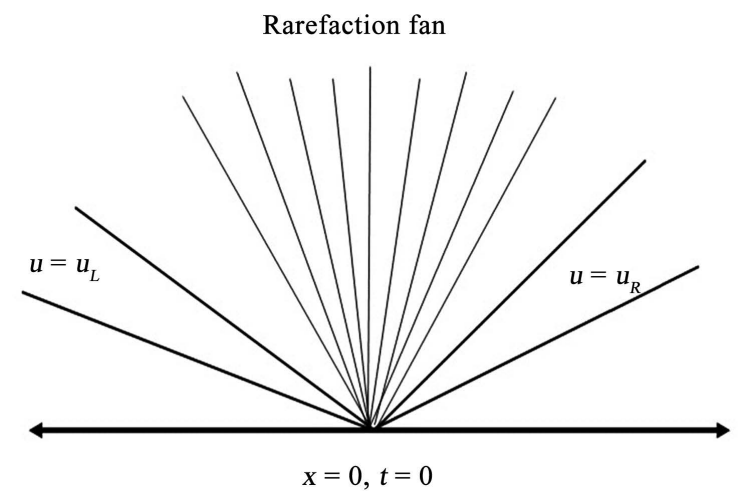

Figure 2. Characteristic curves for rarefaction wave $u_{L}<u_{R}$. 
For simplicity, we have considered the uniform discretization for the special and time domain. Suppose the special domain is $\left[x_{L}, x_{R}\right]$ and then, the discretized point is $x_{i}=x_{L}+(i+1 / 2) \Delta x$ for $i=0,1, \cdots, N$ where $\Delta x=\left(x_{R}-x_{L}\right) / N+1$. Thus, the $i$-th grid cell or control volume is $C_{i}=\left(x_{i-1 / 2}, x_{i+1 / 2}\right)$.

The discretization in time with step size $\Delta t$ is $t^{n}=n \Delta t$.

Now, suppose the value $u_{i}^{n}$ is the cell average over the $i$-th interval at time $t^{n}$. Then,

$$
u_{i}^{n} \approx \frac{1}{\Delta x} \int_{x_{i-1 / 2}}^{x_{i+1 / 2}} u\left(x, t^{n}\right) \mathrm{d} x \equiv \frac{1}{\Delta x} \int_{C_{i}} u\left(x, t^{n}\right) \mathrm{d} x
$$

Similarly, $F_{i+1 / 2}^{n}$ is the average value at the interface $i+1 / 2$ between $t^{n}$ and $t^{n+1}$,

$$
F_{i+1 / 2}^{n}=\frac{1}{\Delta t} \int_{t^{n}}^{t^{n+1}} f\left(u\left(x_{i+1 / 2}, t\right)\right) \mathrm{d} t
$$

Now, integrating the conservation law (1) over $\left[x_{i-1 / 2}, x_{i+1 / 2}\right] \times\left[t^{n}, t^{n+1}\right]$, we get

$$
\int_{t^{n}}^{t^{n+1}} \int_{x_{i-1 / 2}}^{x_{i+1 / 2}} u_{t} \mathrm{~d} x \mathrm{~d} t+\int_{t^{n}}^{t^{n+1}} \int_{x_{i-1 / 2}}^{x_{i+1 / 2}} f(u)_{x} \mathrm{~d} x \mathrm{~d} t=0
$$

Therefore,

$$
\begin{aligned}
& \int_{x_{i-1 / 2}}^{x_{i+1 / 2}} u\left(x, t^{n+1}\right) \mathrm{d} x-\int_{x_{i-1 / 2}}^{x_{i+1 / 2}} u\left(x, t^{n}\right) \mathrm{d} x \\
& =-\int_{t^{n}}^{t^{n+1}} f\left(u\left(x_{i+1 / 2}, t\right)\right) \mathrm{d} t+\int_{t^{n}}^{t^{n+1}} f\left(u\left(x_{i-1 / 2}, t\right)\right) \mathrm{d} t
\end{aligned}
$$

Dividing both side by $\Delta x$, then

$$
u_{i}^{n+1}=u_{i}^{n}-\frac{\Delta t}{\Delta x}\left(F_{i+1 / 2}^{n}-F_{i-1 / 2}^{n}\right)
$$

This equation represents the conservation law directly, that is, the rate of change of the cell average is computed by the flux differences through the boundary of the cell. Therefore, this equation is the exact form of conservation law.

In finite volume method, different way of approximation of flux function will give the different methods. Next, the Godunov method is presented by approximating flux function.

\subsection{Godunov Method}

For Godunov method, we need to approximate the numerical flux $F_{i+1 / 2}^{n}$ at each interface $x_{i+1 / 2}$. As the cell average $u_{i}^{n}$ is constant in each cell $C_{i}$ at each time level, it may define at each cell interface $x_{i+1 / 2}$ a Riemann problem,

$$
\begin{gathered}
\frac{\partial u}{\partial t}+\frac{\partial}{\partial x} f(u)=0 \\
u\left(x, t^{n}\right)= \begin{cases}u_{i}^{n} & \text { if } x<x_{i+1 / 2} \\
u_{i+1}^{n} & \text { if } x>x_{i+1 / 2}\end{cases}
\end{gathered}
$$

According to this method, initially, need to reconstruct a piecewise polynomi- 
al function $\tilde{u}^{n}\left(x, t^{n}\right), \forall x$, from the cell average $u_{i}^{n}$. In the simplest case, this is a piecewise constant function that takes $u_{i}^{n}$ in the $i$-th grid cell, i.e. $\tilde{u}^{n}\left(x, t^{n}\right)=u_{i}^{n}, \forall x \in C_{i}$.

Next, after time $\Delta t$, obtain $\tilde{u}^{n}\left(x, t^{n+1}\right)$, with initial data. In this way, need to average this function over every grid cell to calculate new cell averages $u_{i}^{n+1}=\frac{1}{\Delta x} \int_{C_{i}} \tilde{u}^{n}\left(x, t^{n+1}\right) \mathrm{d} x$. The full process is then repeated in the next time step.

Thus, $\tilde{u}^{n}\left(x_{i-1 / 2}, t\right)$ is constant over the time interval $t^{n}<t<t^{n+1}$. As the Riemann problem has similarity solution. So, Riemann problem centered at $x_{i-1 / 2}$ has a similarity solution along $\left(x-x_{i-1 / 2}\right) /\left(t-t^{n}\right)=$ constant. The value along $\left(x-x_{i-1 / 2}\right) / t=0$ gives the value $\tilde{u}^{n}\left(x_{i-1 / 2}, t\right)$. Denote this value by $u_{i-1 / 2}^{*}=u^{*}\left(u_{i-1}^{n}, u_{i}^{n}\right)$. Therefore, the numerical flux $F_{i-1 / 2}^{n}$ become

$$
\begin{aligned}
F_{i-1 / 2}^{n} & =\frac{1}{\Delta t} \int_{t^{n}}^{t^{n+1}} f\left(u_{i-1 / 2}^{*}\right) \mathrm{d} t=\frac{1}{\Delta t} \int_{t^{n}}^{t^{n+1}} f\left(u^{*}\left(u_{i-1}^{n}, u_{i}^{n}\right)\right) \mathrm{d} t \quad \text { (say) } \\
& =f\left(u^{*}\left(u_{i-1}^{n}, u_{i}^{n}\right)\right)=f\left(u_{i-1 / 2}^{*}\right)
\end{aligned}
$$

Therefore, Godunov method is obtained by solving the Riemann problem between states $u_{i-1}^{n}$ and $u_{i}^{n}$ in order to determine the flux $F_{i-1 / 2}^{n}=f\left(u_{i-1 / 2}^{*}\right)$.

Thus, the Godunov flux for conservation law is [5],

$$
F_{i-1 / 2}^{n}= \begin{cases}f\left(u_{i-1}\right) & \text { if } u_{i-1}>q_{s} \text { and } s>0 \\ f\left(u_{i}\right) & \text { if } u_{i}<q_{s} \text { and } s<0 \\ f\left(u_{s}\right) & \text { if } u_{i-1}<q_{s}<u_{i}\end{cases}
$$

Consequently, there are five possible form of Riemann solution and it contains a single shock or rarefaction wave. The solution $u_{i-1 / 2}^{*}$ is either $u_{i}$ if the shock or rarefaction wave moving entirely to the left and on the other hand, $u_{i-1 / 2}^{*}$ is $u_{i-1}$ if the shock or rarefaction wave moving entirely to the right. Moreover, if the rarefaction wave spreads partly to the left and also partly to the right, at this point, the solution $u_{i-1 / 2}^{*}$ is neither $u_{i-1}$ nor $u_{i}$ and the solution will be $u_{s}$, where $u_{s}$ is the unique value for which $f^{\prime}\left(u_{s}\right)=0$ where $u_{i-1}<u_{s}<u_{i}$.

Where $s$ is the shock speed and $s=\frac{f\left(u_{R}\right)-f\left(u_{L}\right)}{u_{R}-u_{L}}$.

The formula can also be written as [5] [14],

$$
F_{i-1 / 2}^{n}= \begin{cases}\min _{u_{i-1}<u<u_{i}} f(u) & \text { if } u_{i-1} \leq u_{i} \\ \max _{u_{i}<u<u_{i-1}} f(u) & \text { if } u_{i} \leq u_{i-1}\end{cases}
$$

Additionally, it guarantees that the solution satisfies the entropy condition. However, the necessary condition for consistency of the numerical flux function is that the wave speed should be bounded [14], i.e. $\max _{i}\left|f^{\prime}\left(u_{i}^{n}\right)\right|<L$, where $L$ is any constant.

Moreover, the CFL condition for the given problem is $\max _{i}\left|f^{\prime}\left(u_{i}^{n}\right)\right| \frac{\Delta t}{\Delta x} \leq 1$.

\subsection{Examples by Godunov Method}

Here, the initial value problem for inviscid Burgers equation using Godunov method will be presented by some examples. Both discontinuous and continuous initial value problems are considered. 


\subsubsection{Discontinuous Initial Value Problems}

Initially considering inviscid Burgers equation with the following discontinuous initial value problem,

$$
\begin{gathered}
\frac{\partial u}{\partial t}+\frac{\partial}{\partial x}\left(\frac{u^{2}}{2}\right)=0 \\
u(x, 0)= \begin{cases}0.6 & \text { if } x \geq 0.5 \\
0.1 & \text { if } x<0.5\end{cases}
\end{gathered}
$$

The initial condition and the numerical solution using Godunov method is presented in Figure 3 and Figure 4. The numerical solution approximated very well. In this problem, computational time is 0.040 section and also $\Delta t=0.0043$ and $\Delta x=0.01$. Figure 4 also shows the wave at different time, where $n$ is represent the number of iteration. The numerical solution does not show any instability. Here it is clear that shock wave propagates with the initial condition.

Again, considering the following discontinuous initial value problem,

$$
\begin{gathered}
\frac{\partial u}{\partial t}+\frac{\partial}{\partial x}\left(\frac{u^{2}}{2}\right)=0 \\
u(x, 0)= \begin{cases}0.2 & \text { if } x \geq 0.5 \\
0.7 & \text { if } x<0.5\end{cases}
\end{gathered}
$$

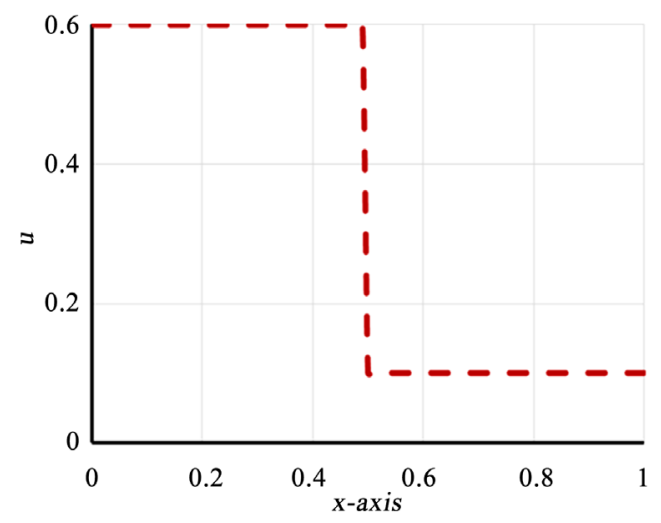

Figure 3. Initial value of (2).

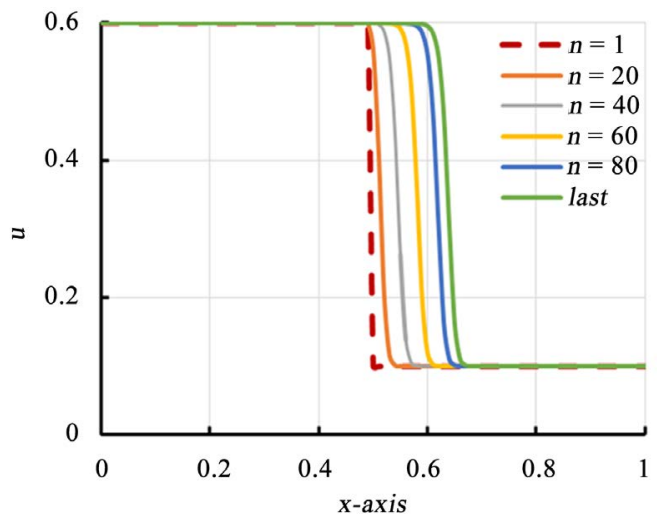

Figure 4. Solution of Burgers equation shows shock wave. 
The initial condition and the numerical solution shows the rarefaction wave using Godunov method is presented in Figure 5 and Figure 6, where, $\Delta t=0.005$ and $\Delta x=0.01$. Figure 6 also shows the wave at different time, where $n$ is represent the number of iteration. The computational time is 0.40 second.

\subsubsection{Continuous Initial Value Problems}

Next, considering inviscid Burgers equation with following continuous initial condition is as smooth as possible. Figures 7-10 show the initial conditions and the numerical solutions by Godunov method of (4) and (5) respectively, where, $x$ - axis is only differ from other.

$$
\begin{gathered}
\frac{\partial u}{\partial t}+\frac{\partial}{\partial x}\left(\frac{u^{2}}{2}\right)=0 \\
u(x, 0)=\sin x, \quad 0 \leq x \leq 2 \pi \\
u(x, 0)=\sin x, \quad 0 \leq x \leq 4 \pi
\end{gathered}
$$

In Figure $8, \Delta t=0.0072$ and $\Delta x=0.011$. In Figure $10, \Delta t=0.0088$ and $\Delta x=0.01$. The computational time is 0.5 second. The speed of propagation of inviscid Burgers equation is $u$ itself. In this case, the initial value moves with variable speed in $x$. As the sine wave is positive at $0 \leq x \leq \pi$, and negative at $\pi \leq x \leq 2 \pi$, the solution is therefore moves first forward and the backward direction, and develop a discontinuity at $x=\pi$.

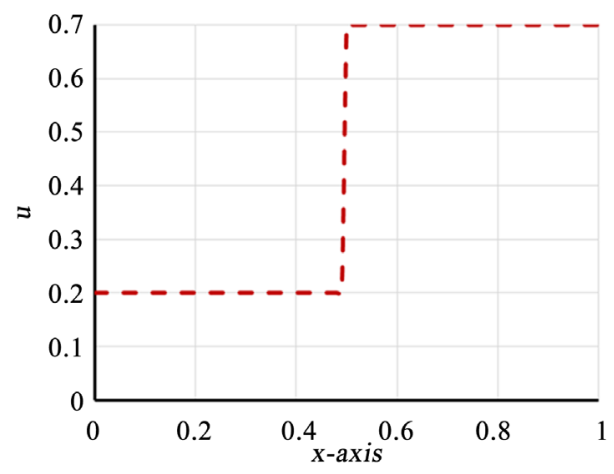

Figure 5. Initial value of (3).

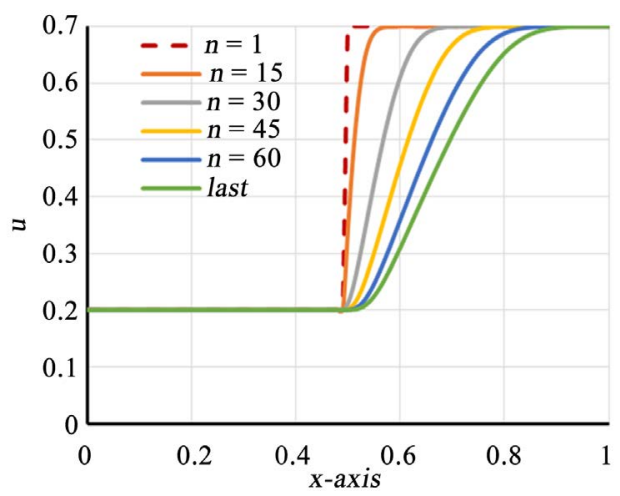

Figure 6. Solution of Burgers equation shows Rarefactions wave. 


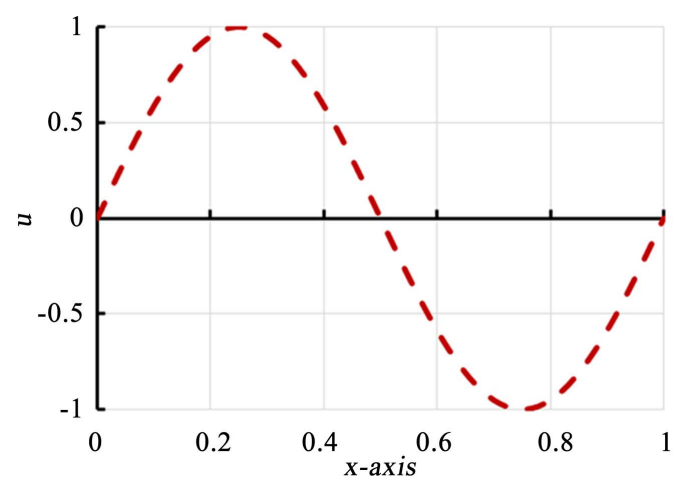

Figure 7. Initial value of (4).

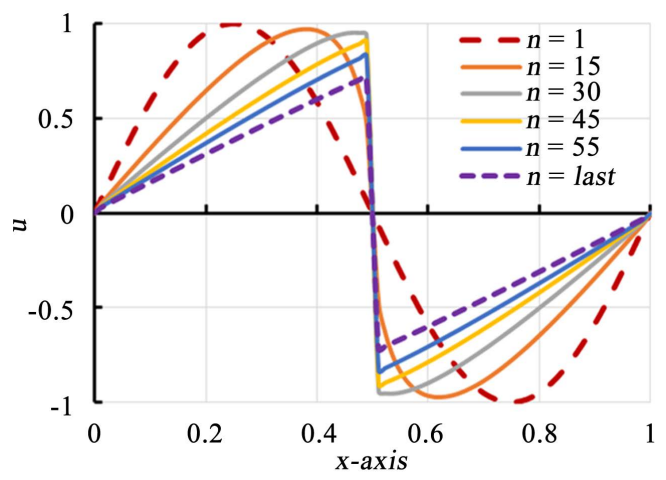

Figure 8. Solution of (4) shows shock wave.

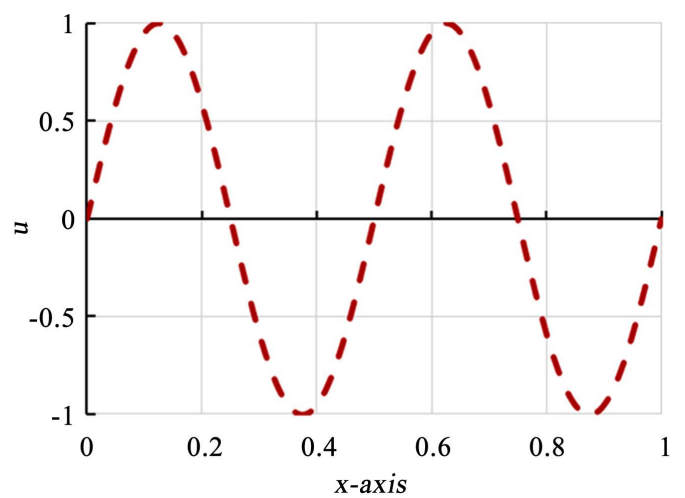

Figure 9. Initial value of (5).

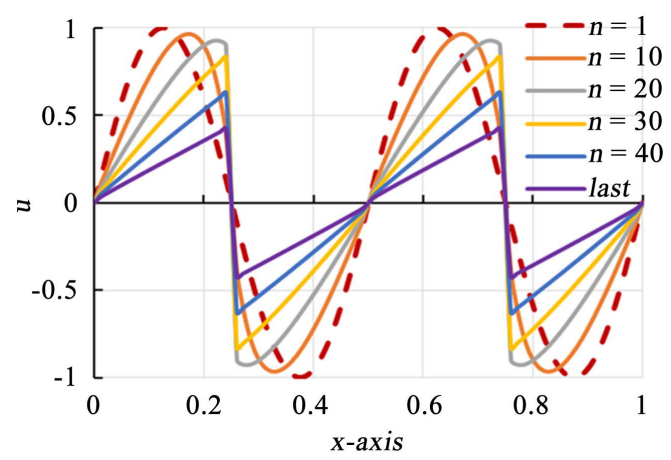

Figure 10. Solution of (5) shows $N$-wave. 
In Godunov method, the solution computed on a very fine mesh. Figure 8 shows initially sine wave compress and develop a shock, and then it expands. In Figure 10, the solution is called $N$-wave also. Therefore, Godunov method shows a good approximation of the solution.

\section{Conclusion}

In this research, inviscid Burgers equation is considered as an example of nonlinear conservation law. Here we presented a theoretical feature of nonlinear conservation law by using the common model, inviscid Burgers equation. Some important features such as integral form of conservation law, classical solution, and weak solution with entropy condition also have been presented. The Riemann problem is discussed to understand the shock wave and rarefaction wave. Finite volume method is considered as numerical method to approximate the solution, as it is effective to describe the discontinuous solution. Taking into account a special initial value problem such as continuous and discontinuous initial value problems are solved by Godunov method. In conclusion, Godunov method shows a good approximation of the problem.

\section{Conflicts of Interest}

The authors declare no conflicts of interest regarding the publication of this paper.

\section{References}

[1] Rugina, C., Bratu, P. and Mosnegutu, V. (2018) On the Bergers Equation with Application to Wave Propagation in Soft Tissue. Romanian Journal of Physics, 3, 37-47.

[2] Yu, L. and Zhou, B. (2014) The Burgers Equation for a New Continuum Model with Consideration of Driver's Forecast Effect. Journal of Applied Mathematics, 2014, Article ID: 539459.https://doi.org/10.1155/2014/539459

[3] Musha, T. and Higuchi, H. (1978) Traffic Current Fluctuation and the Burgers Equation. Japanese Journal of Applied Physics, 17, 811-816. https://doi.org/10.1143/JJAP.17.811

[4] Bonkile, M.P., Awasthi, A., Lakshmi, C., Mukundan, V. and Aswin, V.S. (2018) A Systematic Literature Review of Burgers' Equation with Recent Advances. Pramana, 90, Article No. 69. https://doi.org/10.1007/s12043-018-1559-4

[5] Leveque, R.J. (2004) Finite Volume Methods for Hyperbolic Problems. Cambridge University Press, Cambridge, UK.

[6] Sterck, H.D. and Ullrich, P. (2009) Introduction to Computational PDEs.

[7] Seaïd, M. (2006) Stable Numerical Methods for Conservation Laws with Discontinuous Flux Function. Applied Mathematics and Computation, 175, 383-400. https://doi.org/10.1016/j.amc.2005.07.052

[8] Ucar, Y., and Yagmurlu, N.M. and Tasbozan, O. (2017) Numerical Solutions of the Modified Burgers' Equation by Finite Difference Methods. Applied Mathematics and Computation, 13, 19-30. https://doi.org/10.1515/jamsi-2017-0002

[9] Pandey, K., Verma, L. and Verma, A.K. (2009) On a Finite Difference Scheme for Burgers' Equation. Applied Mathematics and Computation, 215, 2206-2214. 
https://doi.org/10.1016/j.amc.2009.08.018

[10] Karakoç, S.B.G., Başhan, A. and Geyikli, T. (2014) Two Different Methods for Numerical Solution of the Modified Burgers' Equation. The Scientific World Journal, 2014, Article ID: 780269. https://doi.org/10.1155/2014/780269

[11] Justin, D. (2019) Numerical Methods for Conservation Laws. https://jdongg.github.io/post/conservation-laws-1/

[12] Oyar, N. (2017) Inviscid Burgers Equations and Its Numerical Solutions. Thesis, Middle East Technical University, Ankara, Turkey.

[13] Landajuela, M. (2011) Burgers Equation. Becam Report, Basque Center for Applied Mathematics, Bizkaia, Spain.

[14] Mishra, S. (2015) Numerical Methods for Conservation Laws and Related Equations. 